\title{
Garden Plants in Zimbabwe: Their ethnomedicinal uses and reported toxicity
}

\author{
Alfred Maroyi
}

\section{Research}

\begin{abstract}
The present study is aimed at documenting the toxicity and ethnomedicinal uses of garden plants in Zimbabwe. Herbarium and field studies were supplemented by a literature review. Data on ethnomedicinal uses of garden plants in Zimbabwe were assembled together with their poisonous properties. 106 popular garden plant species are regarded as poisonous in Zimbabwe and 23 of these have found various uses in traditional medicine in many parts of the country. They are distributed in 80 genera belonging to 39 families. Families with the highest number of poisonous plants include: Solanaceae (16 species); Euphorbiaceae (14 species); Apocynaceae (9 species) and Fabaceae (9 species). Of these $10.4 \%$ are indigenous and the rest are exotic. Information on these plants is presented together with their toxic and ethnomedical properties, which will serve as a basis for further studies to establish the medicinal claims.
\end{abstract}

\section{Introduction}

An inventory of introduced plants in Zimbabwe was initiated in 1996, for the purpose of documenting exotic plant species which now cover a large proportion of the country's arable land (Maroyi 2006). Garden plants are the basis of human subsistence and cultivated plants are often economically important. The present study focuses on a subset of plants growing in home gardens and houses as food and ornamental plants. Ornamental is used here in a wide sense including all decorative uses, i.e., street trees, hedging, and house plants. Documentation of all categories of plants, whether indigenous or not is important, because as time passes, the distinction between natural and man-made landscapes becomes obscure. Therefore, documentation of cultivated and naturalized plants is essential, as this will assist in the formulation of plant introduction policies. This type of knowledge is also suitable for identification of useful plants that can be targeted for domestication. Domestication of medicinal plants in East Africa, for example, has been seen as a way of increasing income and availability of the products to traditional healers and other resource users (Dery et al. 2000).

Apart from utilizing garden plants for a wide diversity of primary survival and aesthetic purposes, some of them are well-known for their poisonous effects on animals and men. Documentation of plant properties is important for protecting the public from the hazards of possible toxic effects of poisonous plants (Kingsbury 1961), especially food and ornamental plants that we interact with everyday. Most of the plants classified as "poisonous" contain harmful chemicals that can cause discomfort and distress, especially in small children. The term "poisonous plant" designates many kinds of plants as well as a wide range of poisonous or disturbing effects. These effects are generally dermatitis (or skin irritation caused by direct or indirect contact with a plant) and internal poisoning caused by eating plant parts (Hardin \& Arena 1974). Contact der-

\section{Correspondence}

Alfred Maroyi, Biodiversity Department, University of Limpopo, Private Bag X1106, Sovenga 0727, SOUTH AFRICA.

alfred.maroyi@ul.ac.za

Ethnobotany Research \& Applications 10:045-057 (2012)

Published: March 28, 2012 
matitis from irritating plants causes the greatest amount of suffering. Chief among them are Hedera helix L. and Euphorbia spp. (Lewis \& Elvin-Lewis 1977). They are several plant species that cause mild contact dermatitis. Cases of plant poisoning in man may result from an overdose of medicine (Gelfand et al. 1985, Watt \& Breyer-Brandwijk 1962) or stimulant or may be accidental. Poisonings and death from plants are rare as most poisonous plants taste unpleasant and are seldom swallowed. Most plant ingestions occur in young children and are due to berries and flowers of ornamental garden and house plants (Eddleton 2000, Fernando 2001, Joubert 1990, Van Wyk et al. 2002). In adults, poisoning can be either accidental or intentional. Most adult fatalities occur when poisonous plants are mistaken for food, e.g., wild tobacco leaves (Nicotiana glauca R.C. Graham) that are sometimes mistaken for traditional spinach and seeds of Jatropha curcas L. that are mistaken for edible nuts (Van Wyk et al. 2002). Plant poisons in Sri Lanka are commonly ingested with suicidal intent (Fernando 2001), examples include: Nerium oleander L. and Thevetia peruviana (Pers.) K. Schum., which contain numerous toxic cardiac glycosides (Van Wyk et al. 2002). The bulb of Gloriosa superba L., is often ingested accidentally (Fernando 2001), due to its erroneous identification as an edible tuber. Traditional medicines are regarded as a common cause of accidental poisoning (Joubert 1990, Kasilo \& Nhachi 1992, Tagwireyi et al. 2002a,b). Most frequently implicated plant species are J. curcas, Ricinus communis L. and Datura stramonium L. (Huai et al. 2010, Joubert 1990, Tagwireyi et al. 2002a). However, poisoning through traditional medicine occurs when herbal medicine is used outside the safeguards of the traditional knowledge system and due to self-administration (Gelfand et al. 1985, Van Wyk et al. 2002). The high incidences of plant poisonings from traditional medicine are also a consequence of misidentification, incorrect preparation or inappropriate administration and dosage (Reynolds 2005, Stewart \& Steenkamp 2000).

From a medical point of view, many toxic plants have been used for different types of complaints. Lantana camara, for example, has been used in many parts of the world to treat a wide variety of disorders (Morton 1994, Pour et al. 2009, Ross 1999, Sonibare \& Effiong 2008), including cancers and tumours (Ghisalberti 2000, Pour et al. 2009, Sonibare \& Effiong 2008). A tea prepared from the leaves and flowers are taken against fever, influenza and stomach-ache (Ghisalberti 2000, Morton 1994). It has been claimed that a steroid, lancamarone, from the leaves exhibited cardiotonic properties (Sharma \& Kaul 1959) and that lantamine, an alkaloid from the stem bark and roots showed antipyretic and antispasmodic properties comparable to those of quinine (Satri 1962). Strychnos is another genus, not only notorious as a poison, but a well-known traditional herbal medicine. In Africa and Asia, Strychnos has a reputation as a remedy against snakebites and poisonings (Philippe et al. 2004). The reputed emetic and tonic properties play an important part in the use of Strychnos in stomach, abdominal and intestinal complaints as well as in the treatment of worms and parasites (Philippe et al. 2004). Some of these desirable properties of poisonous plants resulted in their domestication as medicinal plants. Poisonous plants, therefore, deserve to be considered important plants when governments are legislating problem plant species. They are a component of plant genetic resources that when protected in agroecosystems, have potentials of becoming useful to man. Already, some of the poisonous plants are not only valuable medicines, but also offer suitable spring boards from which to develop drugs with improved medicinal properties (Ajibesen et al. 2008, Bisset 1991).

However, there is scanty information in Zimbabwe on poisonous plants, particularly the home garden plants that are likely to poison both adults and children. Moreover, other than South Africa, there are only limited and isolated case studies (e.g., Nyazema 1984, Tagwireyi et al. $2002 a, b)$, from the wider human toxicity. Most of the existing literature on poisonous plants in Zimbabwe deals with those plants that are poisonous to livestock (Brain 1934, Collins et al. 1950, Markson 1956, Philip et al. 1958, Shava 2004 , Shone 1959, Shone \& Drummond 1965, Stent \& Lawrence 1932). Many of our plants are poisonous (Watt \& Breyer-Brandwijk 1932) but we lack precise knowledge regarding their effects. Some of the widely utilized food plants, such as Manihot esculenta Crantz, Dioscorea spp. (Bisset 1991, Huai et al. 2010); Trichilia dregeana Sond. (Maroyi 2007a) and medicinal plants, such as D. stramonium (Gelfand et al. 1985, Huai et al. 2010) and R. communis (Huai et al. 2010, Maroyi 2007b), are known to be poisonous. In several tropical countries, the ripe blueblack berries of $L$. camara are eaten, but ingestion of the green berry has led to human fatalities (Ghisalberti 2000, Morton 1994, Ross 1999). This investigation provides additional information on toxicity and ethnomedicinal uses of plants in our home gardens, in some cases on plants on our tables as food and medicines.

\section{Materials and Methods}

In order to initiate a study into the ethnomedicine and toxicology of garden plants in Zimbabwe, an online literature search was conducted using BioMed Central (www. biomedcentral.com), Blackwell Synergy (www.blackwellsynergy.com), CAB Abstracts (www.cabi.org), Elsevier ScienceDirect (www.sciencedirect.com), Ingenta Connect (www.ingentaconnect.com), ISI Web of knowledge (www.isiknowledge.com), intute (www.intute.ac.uk) and JSTOR (www.jstor.org) for scientific articles published before November 2010. References were also identified by searching the extensive library collections of Wageningen University, Rhodes University and the National Herbarium, Harare. These libraries were consulted for books on poisonous plants and ethnomedicinal uses of garden and ornamental plants. Sources of information included: Watt \& Breyer-Brandwijk (1932, 1962); Shone \& Drum- 


\section{Maroyi - Garden Plants in Zimbabwe: Their ethnomedicinal uses and reported toxicity}

mond (1965); Verdcourt \& Trump (1969); Hardin \& Arena (1974); Alpin (1976); Biegel (1977, 1980); Gelfand et al. (1985); Van Wyk et al. 2002; Shava (2004) and Maroyi (2006). Some of the information included here was taken from documents that have accompanied specimens sent to the National Herbarium, Harare, SRGH (abbreviation according to Thiers 2009); for identification over the years. Copies of these documents are filed alphabetically under the name of the plants causing the poisoning and the file is referred to as the "National Herbarium Poisonous Substance File". Specimens of the reported plants were collected in the field. For each species, a voucher specimen is cited and all these specimens are housed at the National Herbarium, Harare (SRGH).

\section{Results}

The results of the survey are presented in Table 1 . The plant species are arranged alphabetically according to family, genus and species. The correct identities and names of all the taxa listed were checked and where names have changed, the currently accepted name was applied. Principal common names are also included (En - English; Sh - Shona and Nd - Ndebele) and these have been included only where the names have been in common use in Zimbabwe. A total of 39 families, 80 genera and 106 plant species are listed. Of these, 11 species are indigenous to Zimbabwe (10.4\%), while 95 species are exotic $(89.6 \%)$, with 35 being naturalized while 60 are confined to home gardens as cultivated plants. The majority of these exotics are found around the homes, offices and in parks either as ornamentals, food plants or weeds of cultivation. Table 1 shows a widespread local knowledge and use of plants by the local people. Out of 106 recorded species, 87 (82\%) have an English, Shona or Ndebele name, although some names are generic and applied to members of the same genus. This may be taken as an indication of local importance, otherwise, there is no need for giving the plants local names if they are not useful to the local community.

\section{Discussion}

\section{Plant toxicity}

While some of the plants in Table 1 are generally recognized as food plants, they may cause a digestive upset and discomfort if eaten, for example, Colocasia esculenta (L.) Schott (Van Wyk et al. 2002); insufficient boiling of hyacinth bean, Lablab purpureus (L.) Sweet (Verdcourt \& Trump 1969), unripe and raw fruits of several garden plants. Some have chemicals called allergens, which may result in dermatitis in some individuals. Of the assessed poisonous plants, 78 species are found in 7 families, rep-

Table 1. Toxic properties of garden plants in Zimbabwe. An asterisk $\left({ }^{*}\right)$ indicates that the taxon is known, or believed, to be naturalized while $\left(^{* *}\right)$ indicates that the taxon is indigenous in Zimbabwe. Language abbreviations: Shona (Sh) and Ndebele (Nd).

\begin{tabular}{|c|c|c|c|}
\hline Plant species & $\begin{array}{l}\text { Voucher } \\
\text { specimen }\end{array}$ & Local name & Toxicity \\
\hline \multicolumn{4}{|l|}{ ANACARDIACEAE } \\
\hline $\begin{array}{l}\text { Anacardium } \\
\text { occidentale L. }\end{array}$ & Ngoni 51 & Cashew nut & $\begin{array}{l}\text { Oily juice from shell irritant to } \\
\text { skin (Senchina 2005) }\end{array}$ \\
\hline *Mangifera indica L. & Noel 224 & Mango & $\begin{array}{l}\text { Toxic principle is allergen urushiol which } \\
\text { cause dermatitis (Senchina 2005) }\end{array}$ \\
\hline Schinus molle L. & $\begin{array}{l}\text { Chipawarasha } \\
400\end{array}$ & California pepper tree & $\begin{array}{l}\text { Plant causes skin irritation } \\
\text { (Van Wyk et al. 2002) }\end{array}$ \\
\hline $\begin{array}{l}\text { Schinus terebinthifolius } \\
\text { Raddi }\end{array}$ & Black 317 & Brazilian pepper tree & $\begin{array}{l}\text { Plant causes skin irritation } \\
\text { (Van Wyk et al. 2002) }\end{array}$ \\
\hline $\begin{array}{l}\text { Smodingium argutum } \\
\text { E. Mey ex Sond. }\end{array}$ & Biegel 4463 & & $\begin{array}{l}\text { Allergen urushiol which cause } \\
\text { dermatitis (Senchina 2005) }\end{array}$ \\
\hline \multicolumn{4}{|l|}{ APIACEAE } \\
\hline${ }^{*}$ Conium maculatum L. & Biegel 4559 & Hemlock & $\begin{array}{l}\text { Y-coniceine causes paralysis of motor } \\
\text { nerve endings (Van Wyk et al. 2002) }\end{array}$ \\
\hline Daucus carota L. & Best 1819 & Carrot & $\begin{array}{l}\text { Falcarinol causes skin irritation } \\
\text { (Van Wyk et al. 2002) }\end{array}$ \\
\hline \multicolumn{4}{|l|}{ APOCYNACEAE } \\
\hline Allamanda cathartica L. & Maroyi 272 & Yellow allamanda & $\begin{array}{l}\text { All parts of the plant cause dermatitis } \\
\text { (Lewis \& Elvin-Lewis 1977) }\end{array}$ \\
\hline
\end{tabular}




\begin{tabular}{|c|c|c|c|}
\hline Plant species & $\begin{array}{l}\text { Voucher } \\
\text { specimen }\end{array}$ & Local name & Toxicity \\
\hline $\begin{array}{l}{ }^{*} \text { Calotropis procera } \\
\text { (Aiton) W.T. Aiton }\end{array}$ & Pope 1650 & Giant milkwood & $\begin{array}{l}\text { Milk latex produces skin inflammation } \\
\text { (Sehgal \& Kumar 2005) }\end{array}$ \\
\hline $\begin{array}{l}{ }^{*} \text { Catharanthus } \\
\text { roseus (L.) G. Don }\end{array}$ & Pope 1364 & Periwinkle & Indole alkaloids (Van Wyk et al. 2002) \\
\hline $\begin{array}{l}\text { Cryptostegia } \\
\text { grandiflora R.Br. }\end{array}$ & Maroyi 284 & Cryptostegia & $\begin{array}{l}\text { Cardiac glycosides interfere with heart } \\
\text { operation (Lewis \& Elvin-Lewis 1977) }\end{array}$ \\
\hline $\begin{array}{l}{ }^{* *} \text { Holarrhena } \\
\text { pubescens Wall. } \\
\text { ex G. Don }\end{array}$ & Maroyi 273 & Fever pod; Muhatsu (Sh) & Alkaloid conessine (Maroyi 2008) \\
\hline Nerium oleander L. & Maroyi 260 & Ceylon rose / Oleander & $\begin{array}{l}\text { Main cardiac glycoside is oleandrin } \\
\text { (Van Wyk et al. 2002) }\end{array}$ \\
\hline Ochrosia elliptica Labill. & Biegel 5551 & & $\begin{array}{l}\text { Fruits are considered poisonous } \\
\text { (Lewis \& Elvin-Lewis 1977) }\end{array}$ \\
\hline Plumeria rubra L. & Biegel 4468 & & $\begin{array}{l}\text { Sap causes dermatitis (Van } \\
\text { Wyk et al. 2002). }\end{array}$ \\
\hline $\begin{array}{l}\text { Thevetia peruviana } \\
\text { (Pers.) K. Schum. }\end{array}$ & Mundy 898 & Yellow oleander & Cardiac glycosides (Van Wyk et al. 2002) \\
\hline \multicolumn{4}{|l|}{ AQUIFOLIACEAE } \\
\hline Ilex aquifolium $\mathrm{L}$. & Biegel 4469 & Holly & $\begin{array}{l}\text { The berry is reputedly harmful } \\
\text { (Van Wyk et al. 2002) }\end{array}$ \\
\hline \multicolumn{4}{|l|}{ ARACEAE } \\
\hline $\begin{array}{l}\text { Alocasia macrorrhizos } \\
\text { (L.) G. Don }\end{array}$ & Maroyi 300 & Giant Elephant's Ear & Oxalates (Van Wyk et al. 2002) \\
\hline $\begin{array}{l}\text { Alocasia odora } \\
\text { (Roxb.) C.Koch }\end{array}$ & Biegel 3398 & Elephant's ear & $\begin{array}{l}\text { Causes skin irritation (Lewis } \\
\text { \& Elvin-Lewis 1977) }\end{array}$ \\
\hline $\begin{array}{l}{ }^{*} \text { Colocasia esculenta } \\
\text { (L.) Schott }\end{array}$ & Mavi 900 & Taro; Mudhumbe (Sh) & $\begin{array}{l}\text { Calcium oxalates cause skin irritation of } \\
\text { the mouth and throat (Van Wyk et al. 2002) }\end{array}$ \\
\hline $\begin{array}{l}\text { Xanthosoma } \\
\text { mafaffa Schott }\end{array}$ & $\begin{array}{l}\text { Goldsmith \& } \\
\text { Müller } 6663\end{array}$ & Yerera (Sh) & $\begin{array}{l}\text { Leaves contain irritant juice } \\
\text { rendering them poisonous } \\
\text { (Lewis \& Elvin-Lewis 1977) }\end{array}$ \\
\hline $\begin{array}{l}\text { Zantedeschia } \\
\text { aethiopica (L.) Spreng. }\end{array}$ & Biegel 5558 & White arum & $\begin{array}{l}\text { Oxalate crystals may cause distress } \\
\text { if eaten (Van Wyk et al. 2002) }\end{array}$ \\
\hline \multicolumn{4}{|l|}{ ARALIACEAE } \\
\hline Hedera helix L. & Biegel 4387 & Ivy & $\begin{array}{l}\text { Contains large amounts of saponins } \\
\text { and skin irritant activity is due to } \\
\text { falcarinol (Van Wyk et al. 2002) }\end{array}$ \\
\hline \multicolumn{4}{|l|}{ ASPARAGACEAE } \\
\hline${ }^{*}$ Agave americana L. & Maroyi 287 & $\begin{array}{l}\text { Agave; Mukonje (Sh); } \\
\text { Isikutsha }(\mathrm{Nd})\end{array}$ & $\begin{array}{l}\text { Sap causes severe skin irritation } \\
\text { (Watt \& Breyer-Brandwijk 1932) }\end{array}$ \\
\hline \multicolumn{4}{|l|}{ ASTERACEAE } \\
\hline Tanacetum vulgare $\mathrm{L}$. & Hopkins s.n. & Tansy & Tinacetin (Van Wyk et al. 2002) \\
\hline${ }^{*}$ Xanthium spinosum L. & Brain 5630 & Spiny cocklebur & $\begin{array}{l}\text { Carboxyatractyloside (Van } \\
\text { Wyk et al. 2002) }\end{array}$ \\
\hline $\begin{array}{l}\text { *Xanthium } \\
\text { strumarium L. }\end{array}$ & Ngoni 384 & Giant cocklebur & $\begin{array}{l}\text { Carboxyatractyloside (Van } \\
\text { Wyk et al. 2002) }\end{array}$ \\
\hline
\end{tabular}


Maroyi - Garden Plants in Zimbabwe: Their ethnomedicinal uses and reported toxicity

\begin{tabular}{|c|c|c|c|}
\hline Plant species & $\begin{array}{l}\text { Voucher } \\
\text { specimen }\end{array}$ & Local name & Toxicity \\
\hline \multicolumn{4}{|l|}{ BIGNONIACEAE } \\
\hline $\begin{array}{l}\text { Campsis radicans (L.) } \\
\text { Seem. ex Bureau }\end{array}$ & Biegel 3698 & Trumpet creeper & $\begin{array}{l}\text { Contact with leaves or flowers cause } \\
\text { dermatitis (Lewis \& Elvin-Lewis 1977) }\end{array}$ \\
\hline \multicolumn{4}{|l|}{ BUXACEAE } \\
\hline Buxus sempervirens $\mathrm{L}$. & Biegel 3479 & Box & Buxine (Lewis \& Elvin-Lewis 1977) \\
\hline \multicolumn{4}{|l|}{ CANNABACEAE } \\
\hline *Cannabis sativa L. & Clark 479 & Dagga; Mbanje (Sh) & $\begin{array}{l}\text { An illegal "narcotic" plant, causes } \\
\text { hallucinations, poor coordination, } \\
\text { stupor and coma (Gray 1975) }\end{array}$ \\
\hline \multicolumn{4}{|l|}{ CAPRIFOLIACEAE } \\
\hline $\begin{array}{l}\text { Sambucus } \\
\text { canadensis L. }\end{array}$ & Simon 2363 & Elderberry & $\begin{array}{l}\text { Leaves, flowers and roots poisonous } \\
\text { (Lewis \& Elvin-Lewis 1977) }\end{array}$ \\
\hline \multicolumn{4}{|l|}{ CARICACEAE } \\
\hline Carica papaya L. & Biegel 3490 & Paw paw; Mupopo (Sh) & $\begin{array}{l}\text { Sap causes skin irritation in some } \\
\text { individuals (Lewis \& Elvin-Lewis 1977) }\end{array}$ \\
\hline \multicolumn{4}{|l|}{ CARYOPHYLLACEAE } \\
\hline Agrostemma githago L. & Nicholls s.n. & Corn - cockle & $\begin{array}{l}\text { Seeds contain toxic githagenin } \\
\text { (Hebestreit \& Melzig 2003) }\end{array}$ \\
\hline \multicolumn{4}{|l|}{ COLCHICACEAE } \\
\hline${ }^{* \star}$ Gloriosa superba L. & Maroyi 243 & $\begin{array}{l}\text { Flame lily; Kajongwe (Sh); } \\
\text { Amakukhulume }(\mathrm{Nd})\end{array}$ & $\begin{array}{l}\text { Extremely poisonous due to } \\
\text { colchicine (Van Wyk et al. 2002) }\end{array}$ \\
\hline \multicolumn{4}{|l|}{ CUCURBITACEAE } \\
\hline $\begin{array}{l}{ }^{*} \text { Cucumis } \\
\text { hirsutus Sond. }\end{array}$ & Rand 30 & Muchacha (Sh) & $\begin{array}{l}\text { Cucurbitacin and saponin } \\
\text { (Van Wyk et al. 2002) }\end{array}$ \\
\hline \multicolumn{4}{|l|}{ CUPPRESSACEAE } \\
\hline Juniperus virginiana L. & Maroyi 280 & Pencil cedar & $\begin{array}{l}\text { Causes allergic rhinitis (Lewis } \\
\text { \& Elvin-Lewis 1977) }\end{array}$ \\
\hline \multicolumn{4}{|l|}{ EUPHORBIACEAE } \\
\hline $\begin{array}{l}\text { Aleurites moluccanus } \\
\text { (L.) Willd. }\end{array}$ & Hodgson $13 / 55$ & Candlenut tree & $\begin{array}{l}\text { Raw seeds are poisonous } \\
\text { (Harborne et al. 1997) }\end{array}$ \\
\hline $\begin{array}{l}{ }^{* *} \text { Croton megalobotrys } \\
\text { Müll. Arg. }\end{array}$ & Maroyi 259 & $\begin{array}{l}\text { Fever-berry croton; } \\
\text { Mushape (Sh) }\end{array}$ & $\begin{array}{l}\text { Seed oil has poisonous diterpenes } \\
\text { (Lewis \& Elvin-Lewis 1977) }\end{array}$ \\
\hline $\begin{array}{l}{ }^{* \star} \text { Euphorbia ingens } \\
\text { E. Mey. ex Boiss. }\end{array}$ & Maroy 279 & $\begin{array}{l}\text { Candelabra tree; } \\
\text { Mukonde (Sh); } \\
\text { Umhlonhlo (Nd) }\end{array}$ & $\begin{array}{l}\text { The plant has irritant and toxic latex, } \\
\text { irritant compound is diterpenoids } \\
\text { (Van Wyk et al. 2002) }\end{array}$ \\
\hline *Euphorbia peplus L. & $\begin{array}{l}\text { Drummond } \\
5524\end{array}$ & & $\begin{array}{l}\text { Latex irritant with diterpenoids } \\
\text { (Van Wyk et al. 2002) }\end{array}$ \\
\hline $\begin{array}{l}\text { Euphorbia pulcherrima } \\
\text { Willd. ex Klotzsch }\end{array}$ & Maroyi 288 & & $\begin{array}{l}\text { Latex irritant with diterpenoids } \\
\text { (Van Wyk et al. 2002) }\end{array}$ \\
\hline $\begin{array}{l}\text { *Euphorbia tulearensis } \\
\text { (Rauh) Rauh }\end{array}$ & Phipps 2415 & $\begin{array}{l}\text { Rubber hedge; Rusungwe } \\
\text { (Sh); Ingotsha (Nd) }\end{array}$ & $\begin{array}{l}\text { Latex irritant with diterpenoids } \\
\text { (Van Wyk et al. 2002) }\end{array}$ \\
\hline Hura crepitans L. & Chase 8111 & Sand box tree & Milky juice poisonous (Macmillan 1989) \\
\hline *Jatropha curcas L. & Maroyi 356 & Physic nut; Mupfuta (Sh) & Curcin (Van Wyk et al. 2002) \\
\hline Jatropha gossypifolia L. & Chase 5680 & & Curcin (Van Wyk et al. 2002) \\
\hline $\begin{array}{l}\text { Jatropha integerrima } \\
\text { Jacq. }\end{array}$ & Maroyi 311 & & Curcin (Van Wyk et al. 2002) \\
\hline
\end{tabular}




\begin{tabular}{|c|c|c|c|}
\hline Plant species & $\begin{array}{l}\text { Voucher } \\
\text { specimen }\end{array}$ & Local name & Toxicity \\
\hline Jatropha multifida L. & Biegel 4763 & Coral tree & Curcin (Van Wyk et al. 2002) \\
\hline $\begin{array}{l}\text { Jatropha podagrica } \\
\text { Hook. }\end{array}$ & Martin 6 & $\begin{array}{l}\text { Bottle plant / } \\
\text { White rhubard }\end{array}$ & Curcin (Lewis \& Elvin-Lewis 1977) \\
\hline $\begin{array}{l}\text { Manihot esculenta } \\
\text { Crantz }\end{array}$ & Biegel 2138 & Cassava; Mufaringa (Sh) & $\begin{array}{l}\text { Cyanogenic glycosides } \\
\text { (Van Wyk et al. 2002) }\end{array}$ \\
\hline${ }^{*}$ Ricinus communis $\mathrm{L}$. & Ward 39 & $\begin{array}{l}\text { Castor oil plant; Mupfuta } \\
\text { (Sh); Umhlafutho (Nd) }\end{array}$ & Ricin and ricinine (Van Wyk et al. 2002) \\
\hline \multicolumn{4}{|l|}{ FABACEAE } \\
\hline $\begin{array}{l}\text { Caesalpinia } \\
\text { gilliesii (Wall. ex } \\
\text { Hook.) D. Dietr. }\end{array}$ & Biegel 3665 & Bird-of Paradise & Poisonous (Van Wyk et al. 2002) \\
\hline $\begin{array}{l}{ }^{* *} \text { Erythrina } \\
\text { abyssinica Lam. }\end{array}$ & Maroyi 320 & $\begin{array}{l}\text { Lucky bean tree; Mutiti } \\
\text { (Sh); Umgqogqogqo (Nd) }\end{array}$ & $\begin{array}{l}\text { Seed poisonous if seed coat } \\
\text { is broken (Shava 2004) }\end{array}$ \\
\hline $\begin{array}{l}\text { *Lablab purpureus } \\
\text { (L.) Sweet }\end{array}$ & Biegel 3975 & $\begin{array}{l}\text { Hyacinth bean; } \\
\text { Chizembera (Sh) }\end{array}$ & $\begin{array}{l}\text { Insufficient boiling of pods may cause } \\
\text { poisoning (Verdcourt \& Trump 1969) }\end{array}$ \\
\hline $\begin{array}{l}\text { Laburnum } \\
\text { anagyroides Medik. }\end{array}$ & Biegel 3476 & & Cytosine (Knight \& Walter 2001) \\
\hline Lathyrus odoratus L. & James s.n. & Sweet pea & $\begin{array}{l}\text { Seeds cause lathyrism which is } \\
\text { due to beta - gamma-L-glutamyl, } \\
\text { aminopropionitrile (Dasler 1954) }\end{array}$ \\
\hline $\begin{array}{l}\text { Leucaena leucocephala } \\
\text { (Lam). De Wit }\end{array}$ & Smith 14 & White popinac & Toxic amino acids (Van Wyk et al. 2002) \\
\hline $\begin{array}{l}\text { ** Mundulea sericea } \\
\text { (Willd.) A. Chev. }\end{array}$ & Maroyi 310 & & Rotenoid (Van Wyk et al. 2002) \\
\hline Phaseolus lunatus L. & Maroyi 324 & Butterbean & $\begin{array}{l}\text { Whole plant contain cyanogenic glycoside } \\
\text { phaseolunatin (Lewis \& Elvin-Lewis 1977) }\end{array}$ \\
\hline $\begin{array}{l}\text { Robinia } \\
\text { pseudoacacia L. }\end{array}$ & Biegel 5805 & False Acacia & $\begin{array}{l}\text { Bark and seeds contain toxins called } \\
\text { robin (Van Wyk et al. 2002) }\end{array}$ \\
\hline \multicolumn{4}{|l|}{ GINKGOACEAE } \\
\hline Ginkgo biloba L. & Biegel 4412 & Maidenhair tree & $\begin{array}{l}\text { Fruit pulp highly irritant to the } \\
\text { skin (Van Wyk et al. 2002) }\end{array}$ \\
\hline \multicolumn{4}{|l|}{ LOGANIACEAE } \\
\hline $\begin{array}{l}\text { ** Strychnos } \\
\text { cocculoides Baker }\end{array}$ & Maroyi 261 & $\begin{array}{l}\text { Monkey orange; } \\
\text { Muzumhwi (Sh) }\end{array}$ & $\begin{array}{l}\text { Strychnine and other indole } \\
\text { alkaloids (Philippe et al. 2004) }\end{array}$ \\
\hline $\begin{array}{l}\text { ** Strychnos } \\
\text { spinosa Lam. }\end{array}$ & Maroyi 299 & $\begin{array}{l}\text { Spiny monkey orange; } \\
\text { Mutamba (Sh) }\end{array}$ & $\begin{array}{l}\text { Strychnine and other indole } \\
\text { alkaloids (Philippe et al. 2004) }\end{array}$ \\
\hline \multicolumn{4}{|l|}{ MELIACEAE } \\
\hline *Melia azedarach L. & Rodin 4422 & $\begin{array}{l}\text { Persian lilac; Musiringa } \\
\text { (Sh); Umsiringa (Nd) }\end{array}$ & $\begin{array}{l}\text { Fruits poisonous due to toxic } \\
\text { limonoids (Van Wyk et al. 2002) }\end{array}$ \\
\hline $\begin{array}{l}\text { ** Trichilia dregeana } \\
\text { Sond. }\end{array}$ & Maroyi 275 & $\begin{array}{l}\text { White mahogany; } \\
\text { Muchichiri (Sh) }\end{array}$ & Bark poisonous (Maroyi 2007a) \\
\hline${ }^{* *}$ Trichilia emetica Vahl. & Maroyi 259 & $\begin{array}{l}\text { Natal mahogany; } \\
\text { Muchichiri (Sh) }\end{array}$ & $\begin{array}{l}\text { Seeds poisonous (Mashungwa } \\
\& \text { Mmolotsi 2007) }\end{array}$ \\
\hline \multicolumn{4}{|l|}{ MELIANTHACEAE } \\
\hline Melianthus major L. & Biegel 5334 & Honey bush & $\begin{array}{l}\text { Root poisonous from glycosides } \\
\text { (Van Wyk et al. 2002) }\end{array}$ \\
\hline \multicolumn{4}{|l|}{ MORACEAE } \\
\hline
\end{tabular}


Maroyi - Garden Plants in Zimbabwe: Their ethnomedicinal uses and reported toxicity

\begin{tabular}{|c|c|c|c|}
\hline Plant species & $\begin{array}{l}\text { Voucher } \\
\text { specimen }\end{array}$ & Local name & Toxicity \\
\hline $\begin{array}{l}\text { Maclura pomifera } \\
\text { (Raf.) C.K. Schneid. }\end{array}$ & $\begin{array}{l}\text { Goldsmith } \\
23 / 69\end{array}$ & Osage orange & $\begin{array}{l}\text { Milky juice causes dermatitis } \\
\text { (Lewis \& Elvin-Lewis 1977) }\end{array}$ \\
\hline \multicolumn{4}{|l|}{ MYRTACEAE } \\
\hline $\begin{array}{l}\text { Melaleuca } \\
\text { quinquenervia } \\
\text { (Cav.) S.T. Blake }\end{array}$ & Müller 578 & Cajeput tree & $\begin{array}{l}\text { Cineole causes dermatitis } \\
\text { (Lewis \& Elvin-Lewis 1977) }\end{array}$ \\
\hline \multicolumn{4}{|l|}{ NYCTAGINACEAE } \\
\hline *Mirabilis jalapa L. & Meara 93 & & $\begin{array}{l}\text { Plant causes gastroenteris in children } \\
\text { (Lewis \& Elvin-Lewis 1977) }\end{array}$ \\
\hline \multicolumn{4}{|l|}{ OLEACEAE } \\
\hline $\begin{array}{l}{ }^{*} \text { Ligustrum lucidum } \\
\text { W.T. Aiton }\end{array}$ & West 6166 & Large - leaved privet & $\begin{array}{l}\text { Fruits contain secoiridoid glycosides } \\
\text { (Van Wyk et al. 2002) }\end{array}$ \\
\hline \multicolumn{4}{|l|}{ OXALIDACEAE } \\
\hline${ }^{*}$ Oxalis latifolia Kunth & Kennan 243 & Purple Garden Sorrel & $\begin{array}{l}\text { Oxalic acid is fatal if excess amounts } \\
\text { are consumed (Van Wyk et al. 2002) }\end{array}$ \\
\hline \multicolumn{4}{|l|}{ PAPAVERACEAE } \\
\hline $\begin{array}{l}{ }^{*} \text { Argemone } \\
\text { mexicana L. }\end{array}$ & Thompson 644 & Mexican poppy & $\begin{array}{l}\text { Whole plant contains isoquinoline } \\
\text { alkaloids (Van Wyk et al. 2002) }\end{array}$ \\
\hline \multicolumn{4}{|l|}{ PHYTOLACCACEAE } \\
\hline Phytolacca dioica L. & Whellan 2048 & Belombra & Triterpenoids (Van Wyk et al. 2002) \\
\hline${ }^{*}$ Phytolacca octandra L. & Simon 768 & Belombra & Poisonous (Lewis \& Elvin-Lewis 1977) \\
\hline \multicolumn{4}{|l|}{ PLUMBAGINACEAE } \\
\hline $\begin{array}{l}\text { Plumbago } \\
\text { auriculata Lam. }\end{array}$ & Brain 5406 & & $\begin{array}{l}\text { Plumbagin causes dermatitis } \\
\text { (de Ruijter 2006) }\end{array}$ \\
\hline \multicolumn{4}{|l|}{ POLYGALACEAE } \\
\hline $\begin{array}{l}\text { **Securidaca } \\
\text { longipedunculata Fres. }\end{array}$ & Maroyi 262 & $\begin{array}{l}\text { Violet tree; Mufufu } \\
\text { (Sh); Umfumfu (Nd) }\end{array}$ & Saponins (Lewis \& Elvin-Lewis 1977) \\
\hline \multicolumn{4}{|l|}{ POLYGONACEAE } \\
\hline $\begin{array}{l}\text { *Fagopyrum } \\
\text { esculentum Moench. }\end{array}$ & $\begin{array}{l}\text { Norlindh \& } \\
\text { Wimarck } 4045\end{array}$ & Buck wheat & $\begin{array}{l}\text { Whole plant causes photosensitization } \\
\text { (Connor 1977) }\end{array}$ \\
\hline${ }^{*}$ Rumex acetosella L. & Eyles 8477 & & $\begin{array}{l}\text { Whole plant has oxalic acid. Toxic if eaten } \\
\text { in large quantities (Van Wyk et al. 2002) }\end{array}$ \\
\hline \multicolumn{4}{|l|}{ PRIMULACEAE } \\
\hline${ }^{*}$ Anagallis arvensis $\mathrm{L}$. & Grosvenor 24 & & $\begin{array}{l}\text { Triterpenoids poisonous if ingested and } \\
\text { cause skin allergies (Van Wyk et al. 2002) }\end{array}$ \\
\hline $\begin{array}{l}{ }^{*} \text { Primula malacoides } \\
\text { Franch. }\end{array}$ & Biegel 2019 & Fairy primula & $\begin{array}{l}\text { Plant causes dermatitis } \\
\text { (Van Wyk et al. 2002) }\end{array}$ \\
\hline \multicolumn{4}{|l|}{ PROTEACEAE } \\
\hline Grevillea banksii R.Br. & Müller 142 & & $\begin{array}{l}\text { Flowers, fruits and seeds contain } \\
\text { cyanogenic glycosides (Sosef } \\
\text { \& van der Maesen 1997) }\end{array}$ \\
\hline $\begin{array}{l}\text { Grevillea robusta } \\
\text { A. Cunn. ex R.Br. }\end{array}$ & Biegel 5164 & Silky oak & $\begin{array}{l}\text { Skin irritant is grevillol (Van } \\
\text { Wyk et al. 2002) }\end{array}$ \\
\hline \multicolumn{4}{|l|}{ ROSACEAE } \\
\hline Malus pumila Mill. & Maroyi 276 & Apple & $\begin{array}{l}\text { Seeds contain cyanogenic glycosides } \\
\text { (Lewis \& Elvin-Lewis 1977) }\end{array}$ \\
\hline
\end{tabular}




\begin{tabular}{|c|c|c|c|}
\hline Plant species & $\begin{array}{l}\text { Voucher } \\
\text { specimen }\end{array}$ & Local name & Toxicity \\
\hline Prunus armeniaca L. & Biegel 4677 & Apricot & $\begin{array}{l}\text { Cyanogenic glycosides } \\
\text { (Van Wyk et al. 2002) }\end{array}$ \\
\hline Prunus cerasus L. & Chase 6924 & Sour cherry & $\begin{array}{l}\text { Cyanogenic glycosides (Lewis } \\
\text { \& Elvin-Lewis 1977) }\end{array}$ \\
\hline $\begin{array}{l}\text { Prunus persica } \\
\text { (L.) Batsch }\end{array}$ & $\begin{array}{l}\text { Rushworth } \\
1051\end{array}$ & Peach; Mupichisi (Sh) & $\begin{array}{l}\text { Cyanogenic glycosides } \\
\text { (Van Wyk et al. 2002) }\end{array}$ \\
\hline Prunus serotina Ehrh. & Davies 2257 & Wild black cherry & $\begin{array}{l}\text { Cyanogenic glycosides } \\
\text { (Van Wyk et al. 2002) }\end{array}$ \\
\hline \multicolumn{4}{|l|}{ RUTACEAE } \\
\hline $\begin{array}{l}\text { Citrus limon } \\
\text { (L.) Osbeck }\end{array}$ & William 225 & Lemon; Lemoni (Sh) & $\begin{array}{l}\text { Thorn and peels cause skin irritation in } \\
\text { some individuals (Van Wyk et al. 2002) }\end{array}$ \\
\hline \multicolumn{4}{|l|}{ SOLANACEAE } \\
\hline Capsicum annuum L. & & Chillies & $\begin{array}{l}\text { May cause severe pain and } \\
\text { distress (Van Wyk et al. 2002) }\end{array}$ \\
\hline $\begin{array}{l}{ }^{*} \text { Cestrum } \\
\text { aurantiacum Lindl. }\end{array}$ & Maroyi & Yellow cestrum & Diterpenols (Van Wyk et al. 2002) \\
\hline *Datura ferox L. & $\begin{array}{l}\text { Drummond } \\
5994\end{array}$ & Datura & Tropane alkaloids (Van Wyk et al. 2002) \\
\hline${ }^{*}$ Datura inoxia Mill. & Evans 1 & & Tropane alkaloids (Van Wyk et al. 2002) \\
\hline Datura metel L. & Phipps 1275 & Horn-of-plenty & Tropane alkaloids (Van Wyk et al. 2002) \\
\hline${ }^{*}$ Datura stramonium L. & Martinean 824 & $\begin{array}{l}\text { Jimson weed / Thorn } \\
\text { apple; Chowa (Sh) }\end{array}$ & Tropane alkaloids (Van Wyk et al. 2002) \\
\hline $\begin{array}{l}\text { *Lycopersicon } \\
\text { esculentum Mill. }\end{array}$ & Jarman 200 & Tomato & a-solanine (Van Wyk et al. 2002) \\
\hline $\begin{array}{l}\text { *Nicotiana glauca } \\
\text { Graham }\end{array}$ & Davies D193 & Tree tobacco & Poisonous due to nicotine (Connor 1977) \\
\hline *Nicotiana tabacum L. & Maroyi 274 & $\begin{array}{l}\text { Tobacco; Fodya } \\
\text { (Sh); Igwayi (Nd) }\end{array}$ & Nicotine (Connor 1977) \\
\hline *Physalis peruviana L. & Grosvenor 683 & $\begin{array}{l}\text { Cape gooseberry; } \\
\text { Mubheri (Sh) }\end{array}$ & $\begin{array}{l}\text { Main alkaloid is hyoscyamine } \\
\text { (Lewis \& Elvin-Lewis 1977) }\end{array}$ \\
\hline $\begin{array}{l}\text { Solanum } \\
\text { aculeatissimum Jacq. }\end{array}$ & Biegel 3486 & & Steroidal alkaloids (Connor 1977) \\
\hline $\begin{array}{l}\text { Solanum linnaeanum } \\
\text { Hepper \& } \\
\text { P.-M.L. Jaeger }\end{array}$ & Eyles 2151 & & Steroidal alkaloids (Van Wyk et al. 2002) \\
\hline $\begin{array}{l}\text { Solanum mauritianum } \\
\text { Scop. }\end{array}$ & Chase 5806 & Bugweed & $\begin{array}{l}\text { Steroidal alkaloids (Lewis } \\
\text { \& Elvin-Lewis 1977) }\end{array}$ \\
\hline Solanum melongena $\mathrm{L}$. & Mahlunge 1 & Egg plant & Steroidal alkaloids (Connor 1977) \\
\hline $\begin{array}{l}\text { Solanum } \\
\text { pseudocapsicum L. }\end{array}$ & Maroyi 64 & Jerusalem cherry & Steroidal alkaloids (Van Wyk et al. 2002) \\
\hline Solanum tuberosum L. & Maroyi 270 & Potato & a-solanine (Van Wyk et al. 2002) \\
\hline \multicolumn{4}{|l|}{ VERBENACEAE } \\
\hline *Duranta repens L. & $\begin{array}{l}\text { Armitage } \\
185 / 55\end{array}$ & & $\begin{array}{l}\text { Whole plant poisonous (Van } \\
\text { Wyk et al. 2002) }\end{array}$ \\
\hline *Lantana camara L. & Gonde $28 / 73$ & $\begin{array}{l}\text { Cherry pie; Mbarapati } \\
\text { (Sh); Besikhiwa (Nd) }\end{array}$ & $\begin{array}{l}\text { Toxic principle is triterpenoid } \\
\text { (Van Wyk et al. 2002) }\end{array}$ \\
\hline
\end{tabular}




\section{Maroyi - Garden Plants in Zimbabwe: Their ethnomedicinal uses and reported toxicity}

resenting $59.4 \%$ of the entire garden plants in Zimbabwe. The remaining $40.6 \%$ belong to 32 families, with the majority represented by one species each. Seven families with particularly high percentage of toxic plant species are the following: Solanaceae (15.1\%); Euphorbiaceae (13.2\%); Apocynaceae (8.5\%); Fabaceae (8.5\%); Rosaceae (4.5\%); Anacardiaceae (4.5\%) and Araceae (4.5\%). Apart from being a reflection of the worldwide high number of species found in these families, this is also a reflection of the toxic principles in the families. The majority of poisonous plants of Solanaceae family are known to possess a diversity of alkaloids that range from mildly irritating to fatal. One of the most important groups of these compounds is tropane alkaloids, whose toxicity and lethality is well documented (Harborne et al. 1997, Van Wyk et al. 2002, Verdcourt \& Trump 1969). While several members of the Euphorbiaceae family are characterized by a milk latex which contains toxic principles such as diterpenoids, triterpenoids and various alkaloids and flavonoids (Alpin 1976, Hardin \& Arena 1974, Lai et al. 2004, Van Wyk et al. 2002, Verdcourt \& Trump 1969, Watt \& Breyer-Brandwijk 1962). The latex is generally poisonous, it damages the eyes and mucous membranes. Members of the Apocynaceae family are the traditional sources of African arrow poisons (Bisset 1991, Watt \& Breyer-Brandwijk 1962). Most members of this family contain cardiac gycosides which have the effect of slowing down the heart rate (Lewis \& Elvin-Lewis 1977, Van Wyk et al. 2002).

\section{Ethnomedicinal uses of poisonous plants}

Of 106 plants with proven or alleged toxicity potential, $22 \%$ have been used in ethnomedicine in Zimbabwe (Table 2). These poisonous plants are used for a number of diseases, ranging from simple headache or stomachache to complicated conditions like epilepsy and convulsions. This high use of potentially harmful plants is consistent with observations made by Watt and Breyer-Brandwijk (1932) that toxic constituents of some poisonous plants are invaluable medicinal drugs when administered at a low dosage. Different parts of medicinal plants are used as medicines, including roots, bark, stem, leaves and plant sap or latex. Methods of preparation of medicines varied for different plants and nature of disease. These include chewing, crushing of parts in order to squeeze out plant sap or oil to inhaling plant smoke. The medicines are administered as decoctions or infusions and also applied externally, in dressing fresh wounds, snake bites and skin diseases (Table 2). The continued use of garden plants in ethnomedicine is due to the fact that they are within easy reach of the community and as such they remain the best alternative to cope with primary health care needs of the community. This widespread use of the potentially harmful traditional medicines in Zimbabwe could also be attributed to cultural acceptability, efficacy and affordability of traditional medicines when compared with modern drugs.

Available documentation on contemporary ethnomedicinal uses of poisonous plants varies from region to region. For India, and to some extent South Africa (e.g., Satri 1962, Sharma \& Kaul 1959, Van Wyk et al. 2002), ethnopharmacological data is well documented, whereas information is scanty and often vague for the rest of tropical countries. The applications of poisonous plants in ethnomedicine will be a strong base for carrying out pharmacological and phytochemical research against the stated ailments. Plants reported for curing more than two ailments need to be screened against the ailments they are used for. Therefore, it is necessary to asses these plants for phytochemical analyses and ethnopharmacological

Table 2. Reported ethnomedicinal uses of poisonous plants in Zimbabwe. Cited from Gelfand et al. (1985) unless otherwise indicated.

\begin{tabular}{|l|l|l|}
\hline Scientific name & Mode of administration & Medicinal uses \\
\hline Agave americana L. & Fiber used as a talisman & Caput medusae \\
\hline Carica papaya L. & Root decoction taken by mouth & Depressed fontanelle \\
\hline Citrus limon (L.) Osbeck & Root infusion taken by mouth & Aphrodisiac \\
\hline \multirow{2}{*}{$\begin{array}{l}\text { Croton megall. Arg. } \\
\text { Cucumis hirsutus Sond. }\end{array}$} & $\begin{array}{l}\text { Becoction or infusion of fruit } \\
\text { / root taken by mouth }\end{array}$ & Swollen stomach (dropsy), purgative \\
\cline { 2 - 3 } & Bark powder taken by mouth in porridge & Abortifacient, infertility in women \\
\hline \multirow{2}{*}{ Datura stramonium L. } & Infusion of leaves taken by mouth & $\begin{array}{l}\text { Abdominal pains, abortifacient, } \\
\text { constipation, convulsions }\end{array}$ \\
\cline { 2 - 3 } & Leaf smoke inhaled & Abdominal pains \\
\hline \multirow{2}{*}{ Erythrina abyssinica Lam. } & Bark / root infusion taken by mouth & $\begin{array}{l}\text { Abdominal pains, Backache, cough, } \\
\text { diarrhea, venereal diseases, wasting } \\
\text { in infants (Kambizi \& Afoyolan 2001) }\end{array}$ \\
\hline & & Wounds in mouth \\
\cline { 2 - 3 } & Mouth washed with root infusion & Lucky charm \\
\cline { 2 - 3 } & Seed ointment applied on face & \\
\hline
\end{tabular}




\begin{tabular}{|c|c|c|}
\hline Scientific name & Mode of administration & Medicinal uses \\
\hline \multirow{2}{*}{$\begin{array}{l}\text { Euphorbia ingens E. } \\
\text { Mey. ex Boiss. }\end{array}$} & Drop of latex taken by mouth in porridge & Bronchitis, emetic, purgative \\
\hline & Latex smoke inhaled & Asthma \\
\hline $\begin{array}{l}\text { Euphorbia tulearensis } \\
\text { (Rauh) Rauh }\end{array}$ & Root infusion taken by mouth & Gonorrhoea \\
\hline Gloriosa superba L. & $\begin{array}{l}\text { Juice of root tuber applied } \\
\text { on painful tooth }\end{array}$ & Toothache \\
\hline $\begin{array}{l}\text { Holarrhena pubescens } \\
\text { Wall. ex G. Don }\end{array}$ & Root infusion taken by mouth & $\begin{array}{l}\text { Abdominal pains, abortifacient, } \\
\text { aphrodisiac, asthma, constipation, } \\
\text { venereal diseases, infertility in } \\
\text { men and women (Mavi 1996) }\end{array}$ \\
\hline Lantana camara L. & $\begin{array}{l}\text { Head washed with decoction } \\
\text { of whole plant }\end{array}$ & Headache \\
\hline \multirow{2}{*}{$\begin{array}{l}\text { Lycopersicon } \\
\text { esculentum Mill. }\end{array}$} & Leaf infusion dropped into the ear & Earache \\
\hline & Root infusion taken by mouth & Blood in urine (Haematuria) \\
\hline Melia azedarach L. & Leaf infusion / decoction taken by mouth & $\begin{array}{l}\text { Abdominal pains, bile emesis, } \\
\text { gonorrhoea, depressed fontanelle }\end{array}$ \\
\hline $\begin{array}{l}\text { Mundulea sericea } \\
\text { (Willd.) A. Chev. }\end{array}$ & Root infusion taken by mouth & Infertility \\
\hline Nerium oleander L. & Leaf infusion taken by mouth & Venereal diseases \\
\hline \multirow[t]{2}{*}{ Nicotiana tabacum L. } & Leaf / root infusion taken by mouth & Asthma, respiratory problems \\
\hline & Rubbing leaf / root & Warts, wounds \\
\hline \multirow[t]{8}{*}{ Ricinus communis L. } & Tooth washed with root decoction & Toothache \\
\hline & Leaf / root infusion taken by mouth & Bilharziasis, heart pains, pneumonia \\
\hline & Seed oil taken by mouth & Abdominal pains \\
\hline & Seed pulverized & Constipation, diarrhea \\
\hline & $\begin{array}{l}\text { Seed ointment applied to } \\
\text { the affected part }\end{array}$ & $\begin{array}{l}\text { Convulsions, depressed fontalle, } \\
\text { external parasites, measles }\end{array}$ \\
\hline & Leaves burnt, smoke inhaled & Hiccoughs \\
\hline & Leaf sap dropped into eyes & Sore eyes \\
\hline & Seed oil dropped into ear & Earache \\
\hline \multirow[t]{5}{*}{$\begin{array}{l}\text { Securidaca } \\
\text { longipedunculata Fres. }\end{array}$} & Root decoction / infusion taken by mouth & $\begin{array}{l}\text { Abdominal pains, aphrodisiac, bile } \\
\text { emesis, constipation, convulsion, } \\
\text { diarrhea, epilepsy, infertility in women, } \\
\text { pneumonia, tapeworm and hookworm, } \\
\text { tuberculosis, venereal diseases }\end{array}$ \\
\hline & Root applied over bitten part & Snakebites \\
\hline & $\begin{array}{l}\text { Root ointment rubbed on back, } \\
\text { infusion taken by mouth }\end{array}$ & Backache \\
\hline & Root powder sniffed & Headache \\
\hline & Root infusion dropped into eyes & Cataracts \\
\hline $\begin{array}{l}\text { Strychnos cocculoides } \\
\text { Baker, Strychnos } \\
\text { spinosa Lam. }\end{array}$ & $\begin{array}{l}\text { Root decoction / infusion taken } \\
\text { by mouth / unripe fruit }\end{array}$ & $\begin{array}{l}\text { Abdominal pains, aphrodisiac, diarrhea, } \\
\text { emetic, gonorrhea, sore throat, infertility }\end{array}$ \\
\hline $\begin{array}{l}\text { Trichilia dregeana Sond., } \\
\text { Trichilia emetica Vahl }\end{array}$ & Bark infusion taken by mouth / enema & Abortifacient, purgative (Mavi 1996) \\
\hline
\end{tabular}




\section{Maroyi - Garden Plants in Zimbabwe: Their ethnomedicinal uses and reported toxicity}

screenings so as to validate the efficacy of the garden plants as herbal medicines. Further study may contribute to development of important pharmaceutical products for future use. Ethnobotanical surveys have been found to be one of the reliable approaches to drug discovery (Ajibesen et al. 2008), and several active compounds have been discovered from plants on the basis of enthnomedicinal information and used directly as patented drugs. Typical examples include: maprouneacin isolated from Maprounea africana Müll. Arg., used as an antidiabetic agent and artemisinin isolated from Artemisia annua L., used as a potent antimalarial compound (Ajibesen et al. 2008).

Results from this investigation are consistent with other published studies that have shown introduced plants to be vital medicines (e.g., Bennet \& Prance 2000, Heinrich 1998, Prance \& Plana 1998). The role of cultivated and exotic plants has been discussed by many authors who have interpreted their diversity of uses in some cultures as a sign of acculturation (Albuquerque et al. 2007). While other workers have pointed out that garden plants, particularly weeds and invasive plants have taken on important roles in traditional pharmacopoeia throughout the world (Stepp 2004, Stepp \& Moerman 2001). Some of the plants discussed in this study have been used for centuries as traditional medicines and the knowledge accumulated in their utilization over generations will assist in identification and isolation of active principles in medicinal preparations.

\section{Conclusions}

Some of the taxa reported in this study are noted for healing properties in their places of origin and in many countries where they occur. Examples include: $R$. communis, Carica papaya L., Citrus limon (L.) Osbeck and L. camara. These species frequently appear in medicinal plant lists from Africa, Europe, Australia, America and Asia. Therefore, there is need for further research into the scientific validation of the plants listed in Table 1, aimed at developing effective drugs that are non-toxic and affordable to the majority of people living in developing countries. This study has shown that some of the important poisonous plants used in ethnomedicine are weeds, belonging to plant families characterized by certain types and classes of toxic chemical compounds. Examples being Solanaceae, Euphorbiaceae and Apocynaceae. No study of ethnomedicine can be done without some basic knowledge of botanical identification of the species, as there is a relationship between taxonomy and chemical profile. People involved in ethnomedical practices have learned how to deal with poisonous latex, seeds and tubers in taxa like Euphorbia spp., R. communis and G. superba.

\section{Acknowledgements}

I would like to thank the Directors of the National Herbarium, Harare (SRGH), Shelmar Schonland Herbarium
(Rhodes University) and Wageningen University Herbarium (WAG) for permission to use the herbarium specimens and library facilities.

\section{Literature Cited}

Ajibesin, K.K., B.A. Ekpo, D.N. Bala, E.E. Essien \& S.A. Adesanya. 2008. Ethnobotanical survey of Akwa lbom State of Nigeria. Journal of Ethnopharmacology 115:387408.

Albuquerque, U.P., P.M. Medeiros, A.L.S. Almeida, J.M. Monteiro, E.M.F.L. Neto, J.G. Melo \& J.P. Santos. 2007. Medicinal plants of the caatinga (semi-arid) vegetation of NE Brazil: A quantitative approach. Journal of Ethnopharmacology 114:325-354.

Alpin, T.E.H. 1976. Poisonous garden plants and other plants harmful to man in Australia. Western Australian Department of Agriculture. Bulletin 3964.

Bennett, B.C. \& G.T. Prance. 2000. Introduced plants in the indigenous pharmacopoeia of northern South America. Economic Botany 54:90-102.

Biegel, H.M. 1977. Checklist of ornamental plants used in Rhodesian parks and gardens. Rhodesian Agricultural Journal Research Report No. 3. Department of Research and Specialist Services, Salisbury.

Biegel, H.M. 1980. Common and botanical names of garden plants in Zimbabwe. Zimbabwe Agricultural Journal Research Report No. 4. Department of Research and Specialist Services, Harare.

Bisset, N.G. 1991. One man's poison, another man's medicine. Journal Ethnopharmacology 32:71-81.

Brain, C.K. 1934. The weeds and poisonous plants of Southern Rhodesia. Pt. 1. Rhodesian Agricultural Journal 47:106-125.

Collins, W.F., H. Wild \& J.C. Hopkins. 1950. Poisonous plants of the Marandellas District. Rhodesian Agricultural Journal 31:779-791.

Connor, H.E. 1977. The Poisonous Plants in New Zealand. Edited by E.C. Keating, Government Printer, Wellington, New Zealand.

Dasler, W. 1954. Isolation of toxic crystals from sweet peas (Lathyrus odoratus). Science 20:307-308.

de Ruijter, A. 2006. Plumbago auriculata Lam. In PROTA (Plant Resources of Tropical Africa / Resources végétales de l'Afrique tropicale). Edited by G.H. Schmelzer \& A. Gurib-Fakim. Wageningen, Netherlands. < http://database.prota.org/search.htm> Accessed 23 March 2008. 
Dery, B.B., R. Otsyina \& C. Ng'atigwa. 2000. Indigenous Knowledge and Prioritization of Medicinal Trees for Domestication in the Shinyanga Region of Tanzania. Shinyanga Soil Conservation Programme (HASHI) / The World Agroforestry Centre (ICRAF), Nairobi.

Eddleton, M. 2000. Patterns and problems of deliberate self-poisoning in the developing world. QJM: International Journal of Medicine 93:715-731.

Fernando, C. 2001. Poisoning due to Abrus precatorius (jequirity bean). Anaesthesia 56:1178-1180.

Gelfand, M., S. Mavi, R.B. Drummond \& B. Ndemera. 1985. The Traditional Medical Practitioner in Zimbabwe: His principles of practice and pharmacopoeia. Mambo Press, Gweru, Zimbabwe.

Ghisalberti, E.L. 2000. Lantana camara L. (Verbenaceae). Fitoterapia 71:467-486.

Gray, C. 1995. Cannabis - The therapeutic potential. Pharmaceutical Journal 254:771-773.

Harborne, J.B., H. Baxter \& G.P. Moss. 1997. Dictionary of Plant Toxins. John Wiley and Sons, Chichester, United Kingdom.

Hardin, J.W. \& J.M. Arena. 1974. Human Poisoning From Native and Cultivated Plants. Duke University Press, Durham, North Carolina.

Hebestreit, P. \& M.F. Melzig. 2003. Cytotoxic activity of the seeds from Agrostemma githago var. githago. Planta Medica 69:921-925.

Heinrich, M. 1998. Plants as antidiarrhoeals in medicine and diet. Pp. 17-30 in Plants for Food and Medicine. Edited by N.L. Etkin, D.R. Harris, H.D.V. Prendergast \& P.J. Houghton. Royal Botanic Gardens, Kew, United Kingdom.

Huai, H., Q. Dong \& A. Liu. 2010. Ethnomedicinal analysis of toxic plants from five ethnic groups in China. Ethnobotany Research \& Applications 8:169-179.

Joubert, P.H. 1990. Poisoning admission of black South Africans. Journal of Toxicology - Clinical Toxicology 28:8594.

Kambizi, L. \& A.J. Afolayan. 2001. Ethnobotanical study of plants used for treatment of sexually transmitted diseases (njovhera) in Guruve District. Journal of Ethnopharmacology $77: 5-9$.

Kasilo, OM. \& C.F. Nhachi. 1992. The pattern of poisoning from traditional medicines in urban Zimbabwe. South African Medical Journal 82:187-188.
Kingsbury, J.M. 1961. Knowledge of poisonous plants in the United States - Brief history and conclusions. Economic Botany 15:119-130.

Knight, A.P. \& R. Walter. 2001. A Guide to Plant Poisoning of Animals in North America. Teton New Media, Jackson, Wyoming.

Lai, X.Z., Y.B. Yang \& X.L. Shan. 2004. The investigation of Euphorbiaceous medicinal plants in Southern China. Economic Botany 58:S307-S320.

Lewis, W.H. \& M.P.F. Elvin-Lewis. 1977. Medical Botany: Plants affecting man's health. Wiley-Interscience, New York, New York.

Macmillan, H.F. 1989. Handbook of Tropical Plants, Tropical Fruits. Anmol Publishers, New Delhi.

Markson, L.M. 1956. On failing to poison chickens with Senecio squalidus L. Veterinary Record 68:333-334.

Maroyi, A. 2006. A preliminary checklist of naturalized and introduced plants in Zimbabwe. Kirkia 18:177-247.

Maroyi, A. 2007a. Trichilia dregeana Sond. Pp. 169-172 in Plant Resources of Tropical Africa 14, Vegetable Oils. Edited by H.A.M. van der Vossen \& G.S. Mkamilo. PROTA Foundation, Wageningen, Netherlands.

Maroyi, A. 2007b. Ricinus communis L. Pp. 144-150 in Plant Resources of Tropical Africa 14, Vegetable Oils. Edited by H.A.M. van der Vossen \& G.S. Mkamilo. PROTA Foundation, Wageningen, Netherlands.

Maroyi, A. 2008. Holarrhena pubescens Wall. ex G. Don. Pp. 332-335 in Plant Resources of Tropical Africa 11, Medicinal Plants 1. Edited by G.H. Schmelzer \& A. Gurib-Fakim. PROTA Foundation, Wageningen, Netherlands.

Mashungwa, G.N. \& R.M. Mmolotsi. 2007. Trichilia emetica Vahl. In PROTA (Plant Resources of Tropical Africa / Resources végétales de l'Afrique tropicale). Edited by in van der Vossen, H.A.M. \& G.S. Mkamilo. Wageningen, Netherlands. < http://database.prota.org/search.htm> Accessed 5 February 2009.

Mavi, S. 1996. Medicinal plants and their uses in Zimbabwe. Pp 67-73 in Indigenous Knowledge and its uses in Southern Africa. Edited by H. Norman, I. Snyman \& M. Cohen. Human Sciences Research Council, Pretoria.

Morton, J.F. 1994. Lantana, or red sage (Lantana camara L., [Verbenaceae]), notorious weed and popular garden flower; some cases of poisoning in Florida. Economic Botany 48:259-270. 


\section{Maroyi - Garden Plants in Zimbabwe: Their ethnomedicinal uses and reported toxicity}

Nyazema, N.Z. 1984. Poisoning due to traditional remedies. Central African Journal of Medicine 30:80-83.

Philip, J.R., J.J. Jackson \& D.K. Shone. 1958. Sarcostemma viminale poisoning in sheep and cattle. Journal of the South African Veterinary Medical Association 29:319-320.

Phillipe, G., L. Angenot, M. Tits \& M. Frédérich. 2004. About the toxicity of some Strychnos species and their alkaloids. Toxicon 44:405-416.

Pour, B.M., S. Sasidharan, R.N. Jegathambigai \& R. Surash. 2009. Anti-leukemia activity of methanolic extracts of Lantana camara. Pharmacognosy Research 1(5):274-279.

Prance, G.T. \& V. Plana. 1998. The use of alien plants in tropical South American folk medicine. Pp. 185-200 in in Plants for Food and Medicine. Edited by N.L. Etkin, D.R. Harris, H.D.V. Prendergast \& P.J. Houghton. Royal Botanic Gardens, Kew, United Kingdom.

Reynolds, T. 2005. Hemlock alkaloids from Socrates to poison aloes. Phytochemistry 66:199-1406.

Ross, I.A. 1999. Medicinal Plants of the World: Chemical constituents, traditional and modern medicinal uses. Humana Press, Totowa, New Jersey.

Satri, B.N. 1962. The Wealth of India. Volume vi. Council of Scientific and Industrial Research, New Delhi.

Sehgal, R. \& V.L. Kumar. 2005. Calotropis procera latexinduced inflammatory hyperalgesia - effect of antiinfammatory drugs. Mediators of Inflammation 2005(4):216220.

Senchina, D.S. 2005. A critical review of herbal remedies for poison ivy dermatitis. Herbalgram 66:35-48.

Sharma, V.S. \& K.N. Kaul. 1959. Indian 59418. Chemical Abstracts 53:652.

Shava, S. 2004. Poisonous Plants of Zimbabwe. Indigenous Knowledge Series, Harare.

Shone, D.K. 1959. Lantana poisoning of cattle. Rhodesian Agricultural Journal 56:238-239.

Shone, D.K. \& R.B. Drummond. 1965. Poisonous Plants of Rhodesia (reprinted from Rhodesian Agricultural Journal 62(4):1-64) Ministry of Agriculture in collaboration with Natural Resources Board of Rhodesia, Rhodesia.

Sonibare, O.O. \& I. Effiong. 2008. Antibacterial activity and cytotoxicity of essential oil of Lantana camara L. leaves from Nigeria. African Journal of Biotechnology 7 (15):2618-2620.
Sosef, M.S.M. \& L.J.G. van der Maesen. 1997. Grevillea banksii R.Br. Pp. 283-284 in Plant Resources of SouthEast Asia No. 11. Auxillary Plants. Edited by I. Faridah Hanum \& L.J.G. van der Maesen. PROSEA Foundation, Bogor, Indonesia.

Stent, S.M. \& D.A.L. Lawrence. 1932. Poisonous or suspected poisonous plants of Southern Rhodesia. Rhodesian Agricultural Journal 29:15-20.

Stepp, J.R. 2004. The role of weeds as sources of pharmaceuticals. Journal of Ethnopharmacology 92:163-166.

Stepp, J.R. \& D.E. Moerman. 2001. The importance of weeds in ethnopharmacology. Journal of Ethnopharmacology 75:19-23.

Stewart, M.J. \& V. Steenkamp. 2000. Toxicology of African herbal remedies. South African Ethnobotany 1:32-33.

Tagwireyi, D., D.E. Ball \& C.F.B. Nhachi. 2002a. Traditional medicine poisoning in Zimbabwe: Clinical presentation and management. Human \& Experimental Toxicology 21:579-586.

Tagwireyi, D., D.E. Ball \& C.F.B. Nhachi. 2002b. Poisoning in Zimbabwe: A survey of eight major referral hospitals. Journal of Applied Toxicology 22:99-105.

Thiers, B. 2009. Index Herbariorum: A global directory of public herbaria and associated staff. New York Botanical Garden's Virtual Herbarium. http://sweetgum.nybg.org/ih/ accessed on 30 January 2009.

Van Wyk, B.-E., F. Van Heerden \& B. Van Oudtshoorn. 2002. Poisonous Plants of South Africa. Briza Publications, Arcadia, South Africa.

Verdcourt, B. \& E.C. Trump. 1969. Common Poisonous Plants of East Africa. Collins, London.

Watt, J.M. \& M.G. Breyer - Brandwijk. 1932. The Medicinal and Poisonous Plants of Southern Africa. E. Livingstone, Edinburgh, United Kingdom.

Watt, J.M. \& M.G. Breyer - Brandwijk. 1962. The medicinal and poisonous plants of Southern and Eastern Africa: Uses, chemical composition, pharmacological effects and toxicology in man and animals. Second edition. E. Livingstone, Edinburgh, United Kingdom. 
